\title{
Roughness Evaluation Approach for Nonstationary Vehicle Noise Based on Wavelet Packet and Neural Network Techniques
}

\author{
Hui Guo, Yansong Wang, Xiaolan Wang, Ningning Liu and Yarong $\mathbf{L i}$ \\ School of Automotive Engineering, Shanghai University of Engineering Science, Shanghai, 201620, China.
}

(Received 6 April 2017; accepted 2 February 2018)

Based on the wavelet packet decomposition (WPD) and artificial neural network (ANN) methods, this paper presents a new technique for auditory roughness evaluation (ARE) of nonstationary vehicle noise, named WPDANN-ARE model. According to sound transfer and perception by the human auditory system, the noise roughness of a sample vehicle under different conditions of constant speed, acceleration, and braking are calculated. After comparisons by the time-frequency analysis techniques in common use, a WPD model with approximately twentyone critical bands, which is specially designed by considering the auditory perception characteristics of human, is proposed for envelope feature extraction of vehicle noise. Taking the WPD extracted features as inputs and the calculated roughnesses as outputs, a back-propagation ANN with one hidden layer is trained and established for ARE of nonstationary vehicle noises. The verification results show that errors of the time-varying roughness calculated from the WPD-ANN-ARE are below 8.56 percent, which suggest a very good accuracy of the newly proposed ARE model. In applications, the WPD-ANN-ARE can be directly used in ARE of vehicle noises. And the modelling approach presented in this paper may be extended to other sound related fields for sound quality evaluation (SQE) in engineering.

\section{INTRODUCTION}

Currently, traffic noise is a major source of noise pollution especially in urban areas, which has attracted more public attention. ${ }^{1}$ Because of the physiological and psychological effects of noise on drivers, this would increase the risk of traffic accidents. ${ }^{1}$ Considerable research effort has been put in sound quality evaluation (SQE) of vehicle noise in the past few decades. ${ }^{2-4}$ The sound quality of a vehicle, as one of the important indices in ride comfort evaluation, has become a key consideration of car buyers and manufacturers.

For auditory characteristics, it is different in the physics and psychoacoustics, due to the human hearing process. Description of the sound quality is multidimensional. Some psychoacoustic indices, such as A-weighted SPL, loudness, sharpness, roughness, fluctuation strength, tonality, annoyance, pleasantness, etc., were proposed to quantitatively relate sound stimuli to human sensations. ${ }^{5-7}$ In the SQE engineering, it has been found that the A-weighted SPL is not a perfect method because the sound masking effects are ignored. Based on the equal-loudness-contours, Zwicker proposed a methodology for calculating the specific loudness, and thereby the loudness and sharpness models. The Zwicker loudness model has been used in the standard ISO $532 \mathrm{~B},{ }^{8}$ and the sharpness model has not yet been internationally standardized but has been accepted by the German national standard DIN 45692. ${ }^{9}$

Auditory roughness is a complex effect which quantifies the perception of rapid amplitude modulation of a sound signal. The unit of measure is the asper. ${ }^{5}$ One asper is defined as the roughness produced by a $1000 \mathrm{~Hz}$ tone of $60 \mathrm{~dB}$ which is $100 \%$ amplitude modulated at $70 \mathrm{~Hz}$. As an important psychoacoustic parameter, the roughness has been widely studied and discussed. Helmholtz first presented the concept of au- ditory roughness. ${ }^{10}$ Hearing tests showed that the amplitude modulated sounds with modulation frequencies from $20 \mathrm{~Hz}$ to $70 \mathrm{~Hz}$ may generate roughness. ${ }^{11}$ Subsequently, many mathematical models for quantitative calculation of auditory roughness were proposed. Some of them were developed by considering the modulation frequency and index, the excitation envelope and level differential, and the autocorrelations in critical bands of a sound. ${ }^{7,12-14}$ The Aures model has been regarded as a flexible method in auditory roughness calculation. ${ }^{7}$ Thus, the improved versions of the Aures model were developed by considering the effects of phase differences and carrier frequencies in the critical bands. ${ }^{15,16}$ Hoeldrich and Pflueger ${ }^{17}$ developed a roughness model to evaluate vehicle interior noise using parameters that could be adjusted for different modulation parameters. The traditional roughness models mainly differ in the methods used to transform the calculated excitations in the critical bands into the roughness. In practice, these methods are difficult to apply because the excitation level differentials in the critical bands are not easy to obtain from real noise signals.

The psychoacoustical indices have been widely considered in quality evaluation of industrial products. In view of the vehicle noise SQE, Hashimoto ${ }^{18}$ studied a psychoacoustical booming index for both stationary and nonstationary interior noises, which were quantified using a weighting function from the subjective response to sensation level. The rumbling noise of a vehicle below $300 \mathrm{~Hz}$ was investigated by Lee, ${ }^{19}$ who pointed out that the human feeling on the rumbling noise mainly depended on the roughness and loudness indices. Due to the nonlinear perception feature of human auditory system, it is sometimes impossible to find an exact physical model to describe the perception response for all people. Thus, following the human hearing process, some SQE methods based on 
virtual human "ear" and "brain" have been recently proposed for SQE of vehicle noises. The time-frequency analysis algorithms, such as the short-time Fourier transform (STFT), discrete wavelet transform (DWT), wavelet packet analysis (WPA) and the Wigner-Ville distributions (WVD), were introduced into SQE engineering for sound feature extraction (virtual "ear") of the impact and nonstationary vehicle noises. ${ }^{20-22}$ Originally used for signal processing, wavelet techniques have been developed and successfully applied in structure analysis, diagnosis of crack faults and wave propagation in structures. ${ }^{39-44}$ Due to good time-frequency characteristics, the wavelet-based algorithms are usually considered for SQE of both stationary and nonstationary noises.

It has been found that, the wavelet band filters with proper sampling rates can be well matched to the critical filter bank in the human auditory system. To classify sound patterns, some techniques based on the artificial neural network (ANN) and the support vector machine (SVM) methods (virtual "brain") were used for predicting the loudness, sharpness and annoyance indices of vehicle noise. ${ }^{23-25}$ Compared with the loudness and sharpness indices, the roughness of a sound is more difficult in the feature extraction and perception modelling, because there exists hardly any correlation between the subjective impressions of test persons and available roughness parameters. ${ }^{17}$ To recognize the patterns of auditory roughness, the ANN used in the loudness and sharpness predictions needs to be reconstructed and modified. From the previous literatures, it has been found that roughness, which has a big contribution to the perceived annoyance of vehicle noise, cannot be neglected. ${ }^{26}$ Currently, there is not any virtual "ear-brain" based approach for auditory roughness prediction. This paper attempts to solve the above key issues and develop a novel model for roughness evaluation of vehicle noise.

Based on the above discussions, we concluded that the insitu methods for roughness calculations remain controversial and cannot garner enough public acceptances to be used in standards. Thus, it is both necessary and useful to develop a new methodology to calculate the auditory roughness. In this paper, a new roughness model is presented by combining the WPD and ANN techniques and evaluates the vehicle interior noise. The modulation index of a sound signal is used instead of the statistical parameters. The WPD-ANN-ARE model is programmed using Matlab and validated by tests. The conclusion is that the developed method is shown to be both accurate and effective for the ARE of vehicle noise, which is beneficial for acoustical design and improvement in vehicle engineering.

\section{ESTABLISHMENT OF VEHICLE NOISE DATABASE}

Sample vehicle noises with a 10 seconds signal frame were measured with the LMS.testlab data acquisition system at the sampling rate of $44100 \mathrm{~Hz}$. The experimental conditions were elaborately arranged, following the measurement method for vehicle interior noise in the standard ISO 5128. ${ }^{28}$ A dry asphalt four-lane two-way test road was selected. Around the test site, there was no sound reflecting object within 50 meters. The weather was clear with a temperature of $26^{\circ} \mathrm{C}$ with no wind when the test were carried on. Meanwhile, the windows were closed; the air conditioner and all electronic equipment in the vehicles were turned off. Two models of Volkswagen, Lavida

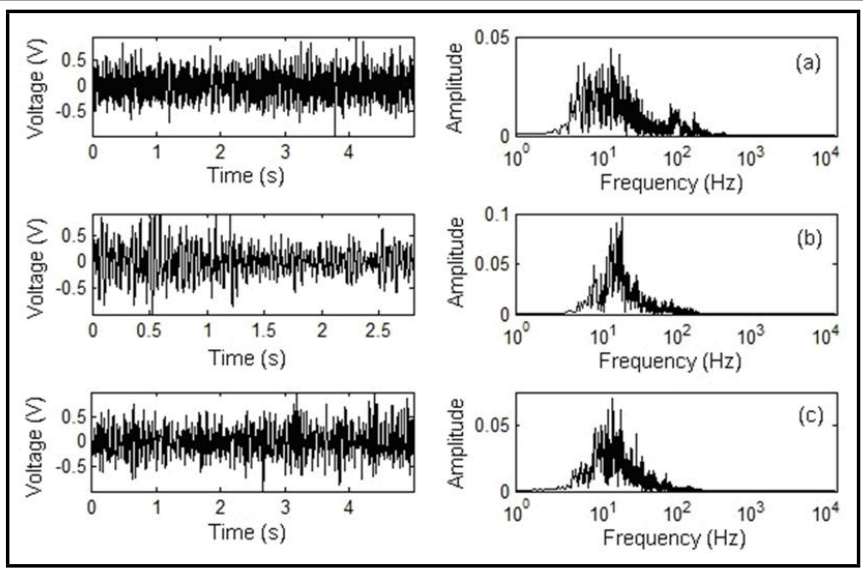

Figure 1. The measured vehicle noise signals and their spectra: (a) acceleration from $50 \mathrm{~km} / \mathrm{h}$ to $120 \mathrm{~km} / \mathrm{h}$, (b) braking from $80 \mathrm{~km} / \mathrm{h}$ to $0 \mathrm{~km} / \mathrm{h}$ and (c) constant speed $70 \mathrm{~km} / \mathrm{h}$.

and Golf, Sedan with 1.6L four-cylinder gasoline engine, were used in the experiments. To meet the requirements of signal number in ARE modelling, eleven vehicle operating conditions were set as constant speed of 30, 40, 50, 60, 70, 80, 90 and $100 \mathrm{~km} / \mathrm{h}$, emergency braking to a stop from 60 and $80 \mathrm{~km} / \mathrm{h}$, and full throttle acceleration from $50 \mathrm{~km} / \mathrm{h}$ to $120 \mathrm{~km} / \mathrm{h}$. Type 4189-A-021 microphones were mounted near to the passenger's right and left ears, according to the GB/T 18697 standard. Under the working conditions, the noise signals were measured and saved in ".mat" and ".wav" formats respectively. The signal-to-noise ratios of the measured signals were above $20 \mathrm{~dB}$, due to well-controlled background noises from the test site and the measurement system. The noise sample at each point with 5 seconds signal frame was selected from the three times measured results by hearing tests. As a preparation for the ARE modelling, a database of the measured interior noises is established. There are 132 noise signal frames (each vehicle: 11 working conditions by 6 channels) of the sample vehicles included in the database, which is used for training and verification of the WPD-ANN-ARE model in the following text.

Figure 1 gives the vehicle noises measured under the working conditions of acceleration from $50 \mathrm{~km} / \mathrm{h}$ to $120 \mathrm{~km} / \mathrm{h}$, braking from $80 \mathrm{~km} / \mathrm{h}$ to $0 \mathrm{~km} / \mathrm{h}$ and constant speed $70 \mathrm{~km} / \mathrm{h}$. It can be seen from their spectra that, regardless of the working conditions, the vehicle noise energy is mainly distributed in a lowfrequency range below $400 \mathrm{~Hz}$. The maximum energy components of the signals are below $50 \mathrm{~Hz}$, which may be caused by vibration of the vehicle body. The energy components between 80 and $150 \mathrm{~Hz}$ are from the second order vibration of the engine. The interior noise energies above $1000 \mathrm{~Hz}$ are very small and can almost be neglected.

\section{AUDITORY ROUGHNESS CALCULATION}

\subsection{Human Auditory-Perception Process}

In psychoacoustics, it has been found that human auditory perception is generated by vibrations from the stapes, which stimulate different parts of the basilar membrane in the inner ear, and thereby the auditory nerve. The auditory perception process with nonlinear filtering properties in the frequency domain can be described by the critical bands. ${ }^{28}$ For a given frequency, the critical band is the smallest band of frequencies around it which activate the same part of the basilar mem- 


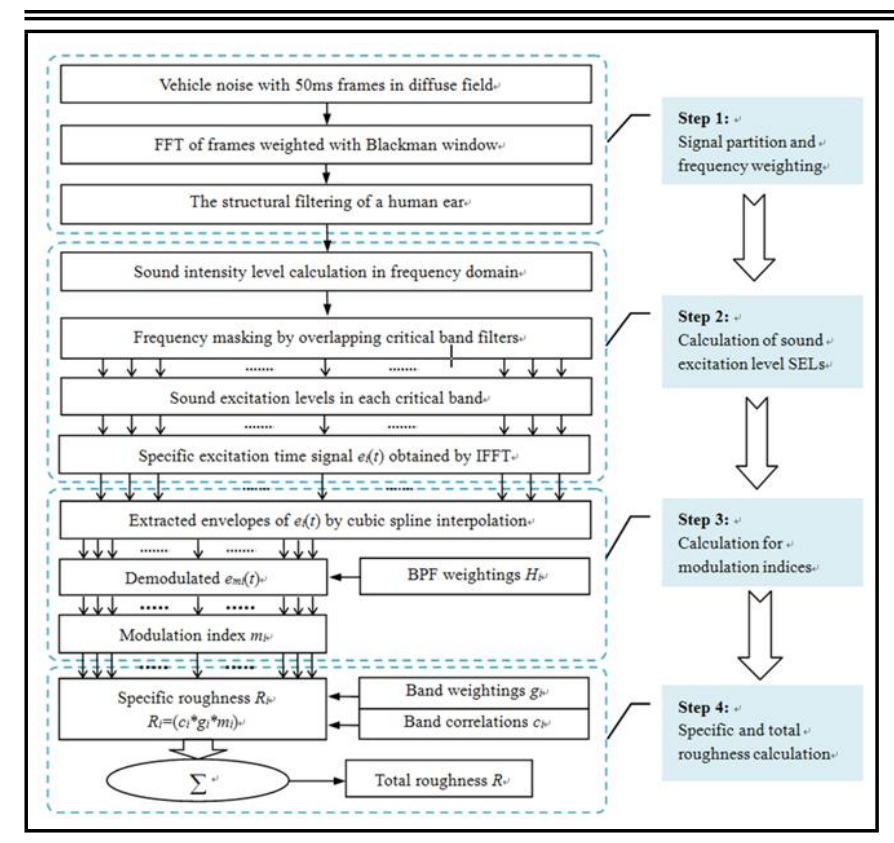

Figure 2. Procedure for roughness calculation of a vehicle noise.

brane. The critical bandwidth represents the human ear's resolving power for simultaneous tones or partials. In this paper, an empirical formula is used to convert one-third octave centre frequency $f c(\mathrm{kHz})$ into critical band rate $z$ (Bark), ${ }^{29}$

$$
z\left(f_{c}\right)= \begin{cases}11.82 \arctan \left(1.21 f_{c}\right) & f_{c} \leq 1.5 \\ 5 \ln \left(f_{c} / 1.5\right)+12.61 & f_{c}>1.5\end{cases}
$$

The critical bandwidths of the Bark scales are defined as $\Delta f_{G}=100 \mathrm{~Hz}$ (if $f_{c}<0.5 \mathrm{kHz}$ ), otherwise $\Delta f_{G}=1 / 5 f_{c}$.

In the SQE field, psychoacoustic parameters such as the weighted SPLs, loudness, sharpness, roughness, fluctuation strength, tonality and articulation index, have been discussed in the past few decades. It has been found that, for a vehicle noise, total effects of the loudness, sharpness and roughness on sound quality are above 90 percent. ${ }^{29}$ This paper focuses only on the study of the roughness index for vehicle noise evaluation.

\subsection{Roughness Index Calculation}

In this paper, an improved Aures model, ${ }^{7}$ which considered the effects of transfer functions of the human ear, modulation index, modulation and carrier frequencies of a signal, is slightly modified and used for auditory roughness calculation. A procedure for roughness computation programming is given in Figure 2. Following the steps in Figure 2, the total roughness of a noise signal can be obtained.

Step 1: Signal partition and frequency weighting. According to the sound masking effects in the time domain, a noise signal is first cut into a set of successive frames with length of 50ms for short-time ARE. The fast Fourier transform (FFT) is performed for calculating the one-third octave SPLs of each frame by using the Blackman window. Considering the structural effects of human ear, the sound transmission coefficients6 are weighted on the signal SPLs, before the signals enter into the inner ear.

Step 2: Calculation of sound excitation level in the Bark domain. The intensity quantity of a sound is based on critical bandwidths and is therefore a subjective representation of frequency. Sound intensity within a critical band can be expressed as

$$
I_{G}(f)=\int_{f-0.5 \Delta f_{G}(f)}^{f+0.5 \Delta f_{G}(f)} \frac{\mathrm{d} I}{\mathrm{~d} f} d f .
$$

Accordingly, sound intensity level (SIL) LG (dB) may be defined as

$$
L_{G}=10 \log \frac{I_{G}(f)}{I_{0}(f)},
$$

where, $f$ is the sound frequency, $I_{0}$ is the intensity of a reference sound, $I_{0}=10^{-12} \mathrm{~W} / \mathrm{m}^{2}$. The maximum SIL is $L_{G}$ in a critical band. The frequency components in each frame are transformed into excitation patterns by overlapping the critical band filters. The calculated sound excitation levels in 24 channels (bands) are transferred into specific excitation time signal $e i(t)$ by the inverse fast Fourier transform (IFFT).

Step 3: Calculation of modulation indices. The cubic spline interpolation method is used for extracting the amplitude envelopes of $e_{i}(t)$. The obtained signal is fed to the weighting function $H_{i}$ of modulation frequency. ${ }^{15}$ The modulated signal emi $(t)$ is obtained. The modulation index in the $i$ th channel $m i$ can be defined as

$$
m_{i}=\frac{r m s_{e m i(t)}}{\left|e_{i}(t)\right|}
$$

where, $r m s_{e m i(t)}$ is the root mean square (RMS) of emi(t), and $\overline{\left|e_{i}(t)\right|}$ is RMS value of the signal $e i(t)$.

Step 4: Specific and total roughness calculations. A phase impact factor ci representing effects of the $(i-1)$ and $(i+1)$ channels on the ith channel is defined as

$$
c_{i}=c_{i-1} \times c_{i+1},
$$

where, $c_{i-1}$ and $c_{i+1}$ are the correlation coefficients of $e_{m i}(t)$ with those in the $(i-1)$ th and $(i+1)$ th channels. Thus, the specific and total values of roughness can be calculated by and in the $i$ th channel may be shown as

$$
\begin{gathered}
R_{i}=\left(g_{i} \times c_{i} \times m_{i}\right)^{2}, \\
R=0.25 \sum_{i=1}^{24} R_{i},
\end{gathered}
$$

where, $R_{i}$ is the specific roughness in the $i$ th channel, $g_{i}$ is the weighting coefficient of carrier frequency, and $R$ is the total roughness.

\section{FEATURE EXTRACTION}

Vehicle noises are typically nonstationary signals, such as the braking or the acceleration noises. Their features should be represented in time and frequency domains. In this paper, the extracted feature matrices of the vehicle noises will be used for ANN training and verification, which needs the matrix spaces to be as small as possible. The frequency spectral analysis and continuous time-frequency representations, such as the continuous wavelet transformm (WT), WVD and its improved versions, cannot satisfy this requirement. ${ }^{21}$ The WPD and Hilbert-Huang Transform (HHT) approaches, which have been frequently mentioned in the fault diagnosis and sound quality fields, ${ }^{20,30,31}$ are considered and compared for feature extraction of the low-frequency acceleration vehicle noise in this paper. 


\subsection{Wavelet Packet Decomposition}

The wavelet transform is the process of decomposing a signal using wavelets. ${ }^{33}$ A family of orthogonal functions as

$$
\Psi_{a, b}(t)=|a|^{-1 / 2} \psi[(t-b) / a] a, b \in R, a \neq 0
$$

are generated from a wavelet function $\psi(t)$ by dilation and translation operations, which are governed by the scale factor $a$ and shift factor $b$. Setting $a=a_{0}^{-j}$ and $b=a_{0}^{-j} k b_{0}$ $\left(j, k \in Z, a_{0}>1, b_{0}>0\right)$, the wavelet function becomes $\psi_{j, k}(t)=a_{0}^{j / 2} \psi\left(a_{0}^{j} t-n b_{0}\right)$. If $a_{0}=2, b_{0}=1$, the discrete wavelet transform (DWT) and its reconstructed version of a signal $x(t) \in L^{2}(R)$ are defined as, ${ }^{33}$

$$
\begin{gathered}
W_{x}(a, b)=W_{x}\left(2^{-j}, 2^{-j} k\right) \\
=2^{j} \int_{-\infty}^{+\infty} \psi\left(2^{j} t-k\right) x(t) d t, \\
x(t)=\sum_{j} \sum_{k} W_{x}\left(2^{-j}, 2^{-j} k\right) \psi\left(2^{j} t-k\right) .
\end{gathered}
$$

Based on the DWT, the WPD was derived using the definitions of the scaling function $\phi(t)$ and the wavelet function $\psi(t)$. Let $u_{0}(t)=\phi(t), u_{0}(t)=\psi(t)$, and define:

$$
\begin{gathered}
u_{2 n}(t)=\sqrt{2} \sum_{k} h_{k} u_{n}(2 t-k), \\
u_{2 n+1}(t)=\sqrt{2} \sum_{k} g_{k} u_{n}(2 t-k)
\end{gathered}
$$

These recursive equations specify a wavelet packet $\left\{u_{n}(t)\right\}$, where $h_{k}$ and $g_{k}$ satisfy the equations $\sum h_{n-2 k} h_{n-2 m}=\delta_{k, m},\left(\sum h_{n}=\sqrt{2}\right), g_{n}=(-1)^{n} h_{1-n}$ and $\left\{u_{j, m, n}(t)=2^{j / 2} u_{m}\left(2^{j} t-m\right)\right\}$, where $j, m$, and $n$ are the scale, translation, and oscillation parameters $j, m \in Z, n \in Z_{+}$. The decomposed coefficients of the signal $x(t)$ is $C^{j, n}=\left\{C_{m}^{j, n}\right\}_{m \in Z}$, where $C_{m}^{j, n}=<x, u_{j, m, n}>$. Thus, WPD of the signal can be obtained by,

$$
\begin{gathered}
C_{m}^{j, 2 n}=\sum_{k=-\infty}^{\infty} h_{2 m-k}^{*} C_{k}^{j+1, n}, \\
C_{m}^{j, 2 n+1}=\sum_{k=-\infty}^{\infty} g_{2 m-k}^{*} C_{k}^{j+1, n} .
\end{gathered}
$$

Conversely, the wavelet packet reconstruction is expressed as the following,

$$
C_{m}^{j+1, n}=\sum_{k=-\infty}^{\infty} h_{2 m-k} C_{k}^{j, 2 n}+\sum_{k=-\infty}^{\infty} g_{2 m-k} C_{k}^{j, 2 n+1}
$$

\subsection{Hilbert-Huang Transform}

The HHT, which is combined by the empirical mode decomposition (EMD) and the Hilbert spectral analysis, is an adaptive time-frequency analysis method for analysing data in nonlinear and nonstationary processes. The HHT kernel is the EMD approach with which a signal can be decomposed into intrinsic mode functions (IMFs). The IMFs yield instantaneous frequencies as functions of time that give an identification of signal components, thereby a signal representation of the HHT in the time and frequency domains. To obtain the instantaneous frequency characteristics of a signal, the IMFs are defined as functions having the same numbers of zero-crossing and extrema and the symmetric envelopes (with respect to time axis) defined by the local maxima and minima. To extract the IMFs from a complex signal in engineering, the EMD needs to be performed. After EMD, the signal $x(t)$ can be expressed as,

$$
x(t)=\sum_{i=1}^{n} c_{i}+r_{n}
$$

where, $c_{i}$ is the ith decomposed IMF of the signal $x(t), r_{n}$ is the residual signal, which occupies very little energy of the signal, can be ignored. Taking the Hilbert transform on both sides of Eq. 16, the Hilbert spectrum $H(\omega, t)$ may be determined by,

$$
H(\omega, t)=\operatorname{Re} \sum_{i=1}^{n} a_{i}(t) e^{j \int \omega_{i}(t) d t}
$$

where, $\mathrm{Re}$ is the operator for the real part, $a_{i}(t)$ and $\omega_{i}(t)$ are the functions of the amplitude and the instantaneous frequency, respectively. $H(\omega, t)$ can describe the signal amplitude varying on a time-frequency plane. The HHT marginal spectrum $h(\omega)$ can be defined as,

$$
h(\omega)=\int_{0}^{T} H(\omega, t) d t
$$

where, $T$ is the length of the signal $x(t), h(\omega)$ reflects the signal amplitude changing with frequency. The instantaneous frequency of the IMFs can localize the signal characteristics in the time-frequency domain.

\subsection{Comparison of WPD and HHT}

As an example, the $50 \mathrm{~km} / \mathrm{h}$ to $120 \mathrm{~km} / \mathrm{h}$ acceleration noise signal was selected to compare the WPD and HHT. As seen in Figure 1, the energy of vehicle noise was concentrated in the low-frequency range below $400 \mathrm{~Hz}$. Thus, for a clearer comparison, the sample signal was first preprocessed by a resampling frequency of $882 \mathrm{~Hz}$, and then filtered by using a high-pass filter with a cutoff frequency of $20 \mathrm{~Hz}$ to match the threshold of the human ear. ${ }^{5}$ A 2level WPD and a 9-level HHT were performed on the preprocessed acceleration noise signal, respectively, using the specifically written Matlab programs. In the WPD, the Daubechies wavelet $d b 35$ was adopted since its characteristics are closer to the critical bands. ${ }^{34}$ In the HHT, the IMFs of the signal were calculated by using the EMD. The decomposed results are shown in Figs. 3 and 4. Figure 3 shows the WPD component signals and their corresponding spectra of the acceleration vehicle noise. Through the 2-level WPD, the original signal was decomposed into four components with approximate averaged frequency bandwidths of $100 \mathrm{~Hz}$, which is very close to the critical bands of human hearing in the low frequencies below $400 \mathrm{~Hz}$. After the HHT, eight IMFs and a residual component of the noise signal were obtained by EMD. The first four IMFs with nearly all energy of the original sound signal and their spectra are shown in Figure 4. It can be seen that the frequency bandwidths of the first, second, third and the fourth IMFs are around 100 to $300 \mathrm{~Hz}, 50$ to $150 \mathrm{~Hz}, 20$ to $50 \mathrm{~Hz}$ and 20 


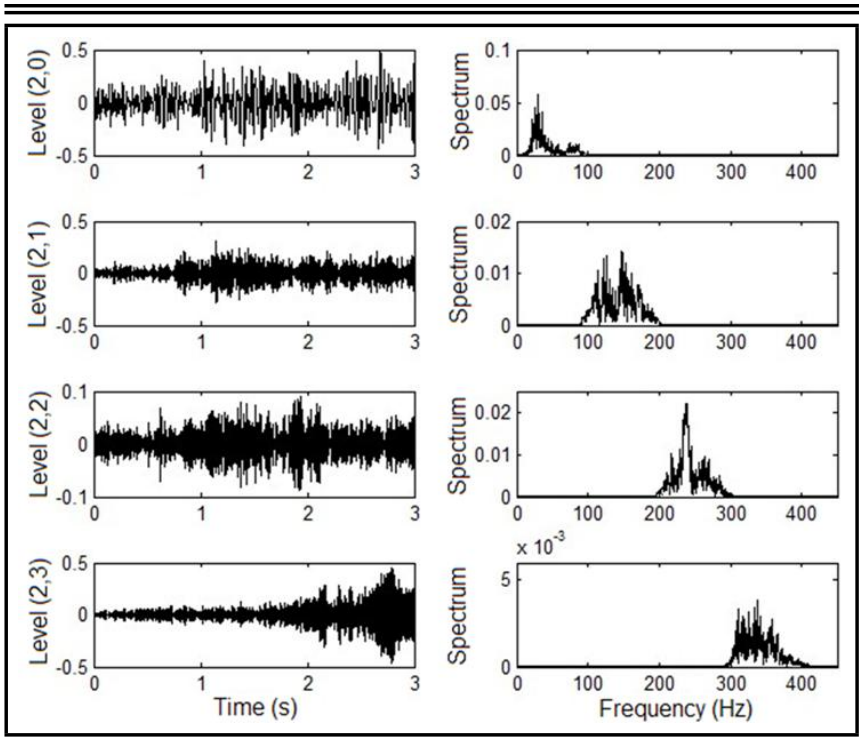

Figure 3. The WPD component signals of a vehicle acceleration noise and their spectra.

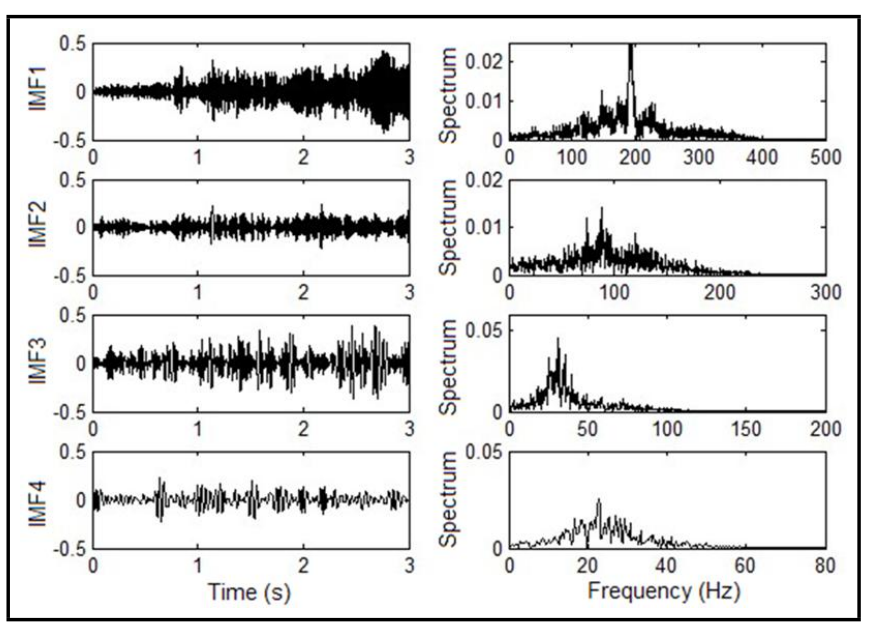

Figure 4. The IMF components calculated by EMD of a vehicle acceleration noise and their spectra.

to $30 \mathrm{~Hz}$, respectively. Frequency overlaps have occurred among the IMF components. It means that the HHT cannot be used to define the critical bands according to the auditory characteristics of human. The EMD is an adaptive method according to the signal features, which led to the random frequency ranges of the IMFs. It can be seen from Figure 3 that, comparing with the HHT, the WPD can decompose a signal into multiple levels and each reconstructed subsignal has a definite relative frequency band. This provides the possibility to specially design for matching the critical bands in the human auditory system. Therefore, the WPD is applied in feature extraction of the nonstationary vehicle noise in this paper.

\subsection{Feature Extraction for Roughness Modelling}

Correct feature extraction of vehicle noise directly affects the accuracy of a sound quality prediction. The feature representation of a sound signal often involves a significant amount of redundancy. To maintain the small size of the network inputs in the following section, it is necessary to reduce the redundant information in the sound feature data in both the time

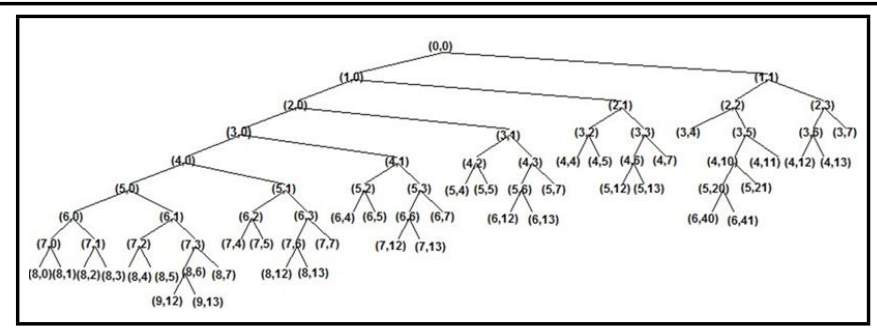

Figure 5. A specially designed wavelet tree for decomposition of vehicle noise signal.

Table 1. Node combinations in the WPD for frequency range approximation to critical bands.

\begin{tabular}{|c|c|c|c|}
\hline $\begin{array}{c}\text { Critical } \\
\text { band rate } z \\
\text { (Bark) }\end{array}$ & $\begin{array}{c}\text { Selected nodes } \\
\text { in the wavelet } \\
\text { tree of WPD }\end{array}$ & $\begin{array}{c}\text { Approximate } \\
\text { frequency ranges } \\
\text { of the WPD }(\mathrm{Hz})\end{array}$ & $\begin{array}{c}\text { Corresponding } \\
\text { frequency ranges } \\
\text { in one-third octave } \\
\text { analysis }(\mathrm{Hz})\end{array}$ \\
\hline 1 & $(8,0)$ & $20-86$ & $22-90$ \\
\hline 2 & $(8,1)$ & $86-172$ & $90-180$ \\
\hline 3 & $(8,2)$ & $172-258$ & $180-280$ \\
\hline 4 & $(8,3)$ & $258-344$ & $280-355$ \\
\hline 5 & $(8,4)$ & $344-430$ & $355-447$ \\
\hline 6 & $(8,5),(9,12)$ & $430-559$ & $447-562$ \\
\hline 7 & $(9,13),(8.7)$ & $559-689$ & $562-708$ \\
\hline 8 & $(7,4)$ & $689-861$ & $708-891$ \\
\hline 9 & $(7,5),(8,12)$ & $861-1119$ & $891-1120$ \\
\hline 10 & $(8,13),(7,7)$ & $1119-1378$ & $1120-1410$ \\
\hline 11 & $(6,4)$ & $1378-1722$ & $1410-1780$ \\
\hline 12 & $(6,5),(7,12)$ & $1722-2239$ & $1780-2240$ \\
\hline 13 & $(7,13),(6,7)$ & $2239-2842$ & $2240-2820$ \\
\hline 14 & $(5,4)$ & $2842-3445$ & $2820-3550$ \\
\hline 15 & $(5,5),(6,12)$ & $3445-4478$ & $3550-4470$ \\
\hline 16 & $(6,13),(5,7)$ & $4478-5512$ & $4470-5620$ \\
\hline 17 & $(4,4)$ & $5512-6890$ & $5620-7080$ \\
\hline 18 & $(4,5),(512)$ & $6890-8957$ & $7080-8910$ \\
\hline 19 & $(5$ 13), $(4,7)$ & $8957-11025$ & $8910-11200$ \\
\hline 20 & $(3,4),(6,40)$ & $11025-14126$ & $11200-14100$ \\
\hline 21 & $\begin{array}{l}(6,41),(5,21) \\
(4,11),(4,12)\end{array}$ & $14126-17916$ & $14100-17800$ \\
\hline
\end{tabular}

and frequency domains. Considering the sound filtering and masking effects of human auditory system, an incomplete 9level WPD model with 21 approximate critical bands was established in the frequency domain and a $50 \mathrm{~ms}$ time interval was selected in the time domain. According to the sampling rate of the signal and the Zwicker model in which the one-third octaves were used instead of the critical bands, the incomplete wavelet tree was specially designed and shown in Figure 5. Except for the nodes $(4,13)$ and $(3,7)$, the end nodes of all WPD levels in the wavelet tree were used to construct the approximate critical bands. The WPD end-node combinations and their corresponding frequency ranges are listed in Table 1.

It should be mentioned that, to simplify the wavelet tree, the measured signals were first resampled at a rate of $S=22050 \mathbf{H z}$ and passed through a high-pass filter with a cutoff frequency of $20 \mathrm{~Hz}$, because hearing threshold of human is from $20 \mathrm{~Hz}$ to $20 \mathrm{kHz}^{5}$ The 9-level WPD with Daubechies wavelet ' $\mathrm{db} 35$ ' with filter length of 70 was performed by the Mallat algorithm. A set of wavelet band-pass filters, combined by low- and high-pass filters with different sampling rates, was created. ${ }^{34}$ Thus, if the WPD nodes were properly combined, the wavelet band-pass filters can be matched to the one-third octaves in the Zwicker model. 
For the $N$-level WPD, a wavelet filter bank with $2^{N}-1$ band-pass filters may be created by the Mallat algorithm, which is combined by $2^{N}$ pairs of low- and high-pass filters. Through the Mallat algorithm, the ' $\mathrm{db} 35$ ' quadrature mirror filters $H, h$ and $G, g$ were used for signal decomposition and reconstruction, where $H, G$ and $h, g$ denote the low- and high-pass filters, respectively. For a sub-signal decomposition at node $(n, m)$ in the wavelet tree, the frequency $(2 m+1) S / 2^{n+1} \mathbf{H z}$ was defined as the upper cutoff frequency of $H$, as well as the lower cutoff frequency of $G$. Inversely, the sub-signal should be reconstructed by the filters $h$ and $g$. The approximate frequency ranges in Table 1 were defined by the cutoff frequencies of the wavelet bandpass filters. For example, the 21th critical band was combined by the nodes $(6,41),(5,21),(4,11)$ and $(4,12)$. The lower and upper frequencies of the wavelet band-pass filter, which consists of the high-pass filter at node $(5,20)$ and the low-pass filter at node $(3,6)$ in Figure 5 , are very close to those of the corresponding one-third octave in the Zwicker model, as seen in Table 1. In the implementation, the resampled signals were fed to the WPD filter bank, which should actually be completed by applying the 9-level WPD to the resampled interior noise. The noise component in the 21th critical band could be finally obtained by summing the reconstructed sub-signals at the related four nodes. The Bark scale serial number is defined as 1 to 24 . The scales (critical bands) 22, 23, and 24 in high frequencies were ignored, since vehicle noise is in a lower frequency range. Considering the sound masking effects of human auditory system in the time domain, very short duration backward masking may be neglected, and the forward masking gradually attenuates in the form of an exponential which approaches zero at $50 \mathrm{~ms} .{ }^{16}$ Accordingly, in this paper, the interval $50 \mathrm{~ms}$ was selected and applied to reduce the redundant information in the time domain sound signal.

Auditory roughness is defined as the human perception to the low-frequency envelope fluctuations of a sound signal. There is a bandpass relationship between the roughness and modulation sound frequency. Thus, the modulation index $m$ was selected as a representation of roughness feature of sounds. A scheme of roughness feature extraction designed for vehicle noises is shown in Figure 6. Firstly, a vehicle noise was decomposed by using the WPD in Figure 5, and the decomposed 21 sub-signals were reconstructed and combined according to the definitions in Table 1 . The 21 sub-signals were numbered by $i$, where $i$ equals $1,2,3 \ldots 21$. In the time dimension, the sub-signals were divided into $R$ frames with a $50 \mathrm{~ms}$ interval. In the $j$ th time interval, following Step 2 in the procedure of roughness calculation in Figure 2, the cubic spline interpolation was used to extract the signal envelopes $e i j(t)$. As an example, the envelope of the first sub-signal of a vehicle noise is shown in Figure 7. The extracted signal envelopes $\operatorname{eij}(t)$ were further filtered by the weighting functions $H_{i}$ of modulation frequency shown in Figure 8, thereby emij $(t)$. Using Eq. 4, the modulation index $m_{i j}$ in the $i$ th band can be calculated. It was finally obtained a roughness feature matrix with a size of 21 by $R$ for each of the noise signals. An example roughness feature matrix is given in Table 2 . It can be seen from the extracted matrix that the roughness feature quantities of a vehicle noise fluctuate with both the time and frequency, and mainly distributes in the low Bark scales.

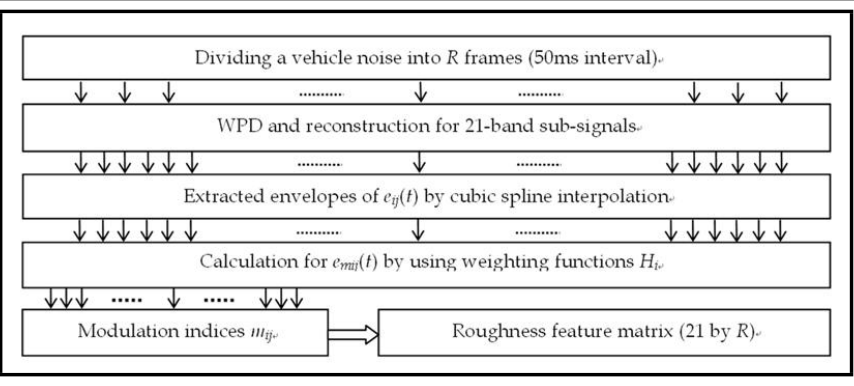

Figure 6. A designed scheme for roughness feature extraction of vehicle noise

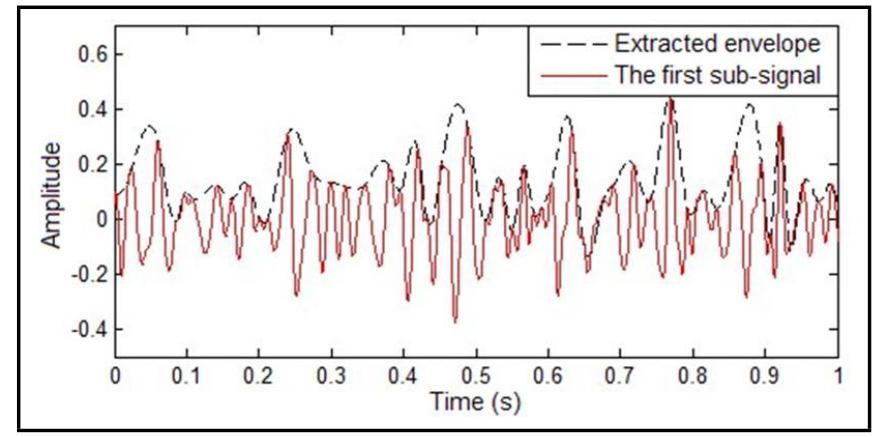

Figure 7. The envelope of the first sub-signal extracted by using the cubic spline interpolation method.

\section{WPD-ANN-ARE MODELING AND VERIFICATION}

\subsection{Artificial Neural Network with Back-Propagation Algorithm}

The ANN, a mathematical model inspired by biological neural networks, is composed of many interconnected artificial neurons operating in parallel. The multilayered feedback network is commonly used in engineering.

The ability to reproduce arbitrary nonlinear functions of input makes them suitable for complex pattern recognition tasks. In this work, a feed-forward ANN with three-layer perceptron was adopted to project the input signal features to the output roughness patterns of vehicle noises, as shown in Figure 9. The inputs are multiplied by related weights and summed, and then passed through a sigmoid function. The sample $x_{k}$ is fed to the network and produces an output $y$. The input pattern $x_{k}$ is

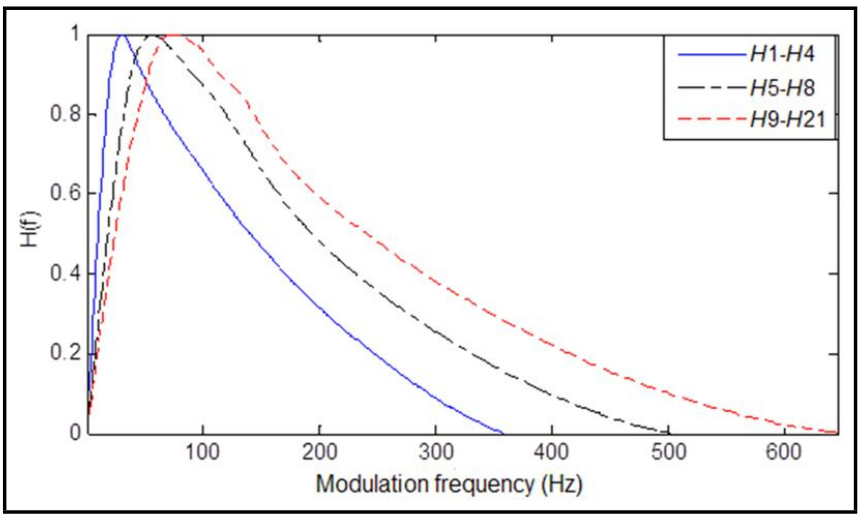

Figure 8. The weighting filters of modulation frequency in different critical bands. 
H. Guo, et al.: ROUGHNESS EVALUATION APPROACH FOR NONSTATIONARY VEHICLE NOISE BASED ON WAVELET PACKET AND...

Table 2. The extracted roughness feature matrix of a vehicle noise in time-frequency domain.

\begin{tabular}{||c|c|c|c|c|c|c|c||}
\hline$z$ (Bark) & $0-50 \mathrm{~ms}$ & $50-100 \mathrm{~ms}$ & $100-150 \mathrm{~ms}$ & $150-200 \mathrm{~ms}$ & $200-250 \mathrm{~ms}$ & $250-300 \mathrm{~ms}$ & $300-350 \mathrm{~ms}$ \\
\hline 1 & 0.534047 & 0.237358 & 0.588959 & 0.519934 & 0.285207 & 0.179029 & 0.295678 \\
\hline 2 & 0.202420 & 0.239060 & 0.347963 & 0.271142 & 0.177252 & 0.180797 & 0.230112 \\
\hline 3 & 0.299086 & 0.297144 & 0.296830 & 0.323990 & 0.285190 & 0.263383 & 0.294479 \\
\hline 4 & 0.152962 & 0.214216 & 0.266093 & 0.260634 & 0.156341 & 0.151784 & 0.187425 \\
\hline 5 & 0.307120 & 0.324465 & 0.207618 & 0.157338 & 0.231361 & 0.231824 & 0.220478 \\
\hline 6 & 0.176511 & 0.264153 & 0.292245 & 0.244347 & 0.255013 & 0.209269 & 0.227302 \\
\hline 7 & 0.231546 & 0.223315 & 0.183365 & 0.162846 & 0.152775 & 0.194528 & 0.220136 \\
\hline 8 & 0.238628 & 0.190091 & 0.165153 & 0.156044 & 0.179326 & 0.164027 & 0.194636 \\
\hline 9 & 0.231651 & 0.219152 & 0.164951 & 0.154834 & 0.252755 & 0.277968 & 0.244536 \\
\hline 10 & 0.268147 & 0.168014 & 0.093789 & 0.135045 & 0.195849 & 0.159032 & 0.136347 \\
\hline 11 & 0.469105 & 0.163835 & 0.158002 & 0.155309 & 0.119621 & 0.160984 & 0.138859 \\
\hline 12 & 0.257936 & 0.139854 & 0.186358 & 0.243770 & 0.187239 & 0.133022 & 0.159618 \\
\hline 13 & 0.191223 & 0.122869 & 0.135978 & 0.174366 & 0.192444 & 0.219321 & 0.156835 \\
\hline 14 & 0.239072 & 0.096717 & 0.066766 & 0.085774 & 0.100432 & 0.093885 & 0.082957 \\
\hline 15 & 0.280601 & 0.139310 & 0.131546 & 0.097453 & 0.085003 & 0.089809 & 0.104920 \\
\hline 16 & 0.326468 & 0.105770 & 0.115106 & 0.184955 & 0.171456 & 0.105484 & 0.111367 \\
\hline 17 & 0.154201 & 0.087618 & 0.092174 & 0.107216 & 0.097012 & 0.089157 & 0.063544 \\
\hline 18 & 0.243163 & 0.092721 & 0.081257 & 0.075249 & 0.077262 & 0.092905 & 0.125949 \\
\hline 19 & 0.230395 & 0.085125 & 0.102897 & 0.106736 & 0.085199 & 0.089744 & 0.091888 \\
\hline 20 & 0.286989 & 0.136321 & 0.196382 & 0.153535 & 0.130648 & 0.139838 & 0.111542 \\
\hline 21 & 0.105753 & 0.064323 & 0.059713 & 0.097012 & 0.111512 & 0.077587 & 0.078394 \\
\hline
\end{tabular}

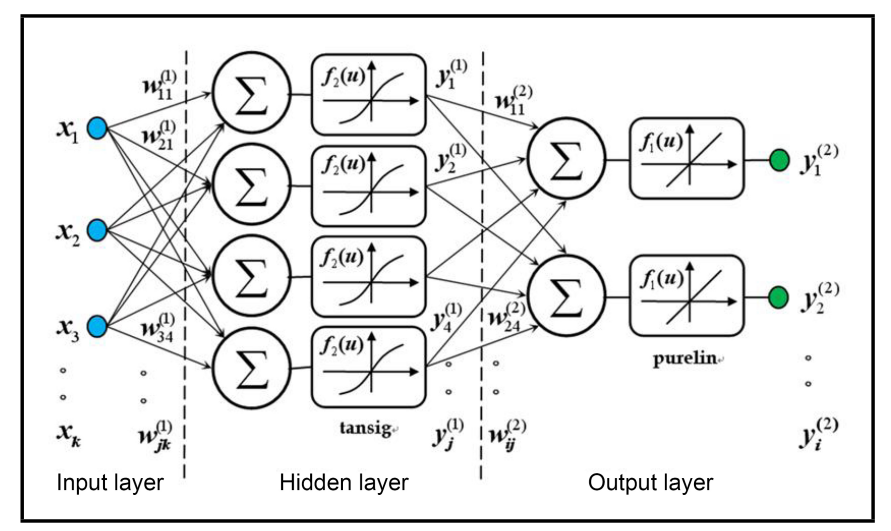

Figure 9. A three-layer BP neural network with a hidden layer.

propagated through the network in the following way:

$$
\begin{aligned}
& y_{i}^{(2)}=f_{1}\left(\sum_{j=1}^{M} w_{i j}^{(2)} y_{j}^{(1)}\right) \\
& =f_{1}\left(\sum_{j=1}^{M} w_{i j}^{(2)} f_{2}\left(\sum_{k=1}^{N} w_{i j}^{(1)} x_{k}\right)\right),
\end{aligned}
$$

where, $y_{j}^{(1)}$ and $y_{i}^{(2)}$ are the outputs of the hidden and output units, and $N$ and $M$ are the numbers of input and hidden units; $w_{i j}^{(n)}$ is the weight corresponding to the $j$ th source, $i$ th target, and $n$th layer; $f_{1}$ and $f_{2}$ are the transfer functions for the hidden and output layers, respectively. The pure linear (purelin) and hyperbolic tangent (tansig) transfer functions are selected in this paper, which are defined as,

$$
f_{1}(u)=u, f_{2}(u)=\frac{2}{1+e^{-2 u}}-1 .
$$

Thus, the tansig and purelin transfer functions were selected according to the empirical formula. ${ }^{35}$

The back-propagation (BP) algorithm is a gradient-search approach that attempts to minimize the squared error of the output by adjusting the weights in the network backwards. The mean squared error (MSE) of BP can be described as,

$$
E=\frac{1}{2|L|} \sum_{i=1}^{|L|} \sum_{\{x\} \in L}[d(\{x\})-y(\{x\})]^{2},
$$

where, $d(x)$ is the desired network output for sample $x$, and $|L|$ is the cardinality of a learning set. To minimize $E$, the change in weight for output unit $i$ from hidden unit $j$ can be derived by the gradient,

$$
\begin{aligned}
& \Delta w_{i j}^{(2)}=\frac{\partial E}{\partial w_{i j}^{(2)}} \\
& =\sum_{\{x\} \in L}\left[d_{i}-y_{i}^{(2)}\right] f\left(y_{i}^{(2)}\right)\left(1-f\left(y_{i}^{(2)}\right)\right) y_{j}^{(1)} .
\end{aligned}
$$

By the chain rule, the weight change for hidden units can be given by,

$$
\begin{aligned}
& \Delta w_{j k}^{(1)}=\sum_{\{x\} \in L} \frac{\partial E}{\partial y_{j}^{(1)}} \frac{\partial y_{j}^{(1)}}{\partial w_{j k}^{(1)}} \\
& =\sum_{\{x\} \in L} \sum_{i} \delta_{i}^{(2)} w_{i j}^{(2)} f\left(y_{j}^{(1)}\right)\left\{x_{k}\right\}
\end{aligned}
$$

in which, $\delta_{i}^{(2)}$ is back-propagated to the hidden layers, $\delta_{j}^{(2)}=$ $\left\lfloor d_{i}-y_{i}^{(2)}\right\rfloor f\left(y_{i}^{(2)}\right)$. Using the momentum adaptive-learningrate algorithm, ${ }^{35}$ the weight can be updated by $w_{\text {new }}=(1-$ $\alpha) w_{\text {old }}-\eta \Delta w$, where $\eta$ and $\alpha$ denote the learning rate and the momentum term, respectively.

\subsection{Architecture of WPD-ANN-ARE Model}

An established three-layer ANN model for ARE is shown in Figure 9. However, some parameters for performing the ANN, such as neuron (node) numbers of the input, hidden and output layers, and the network training algorithm, need to be specified. The extracted roughness feature vector within a $50 \mathrm{~ms}$ 


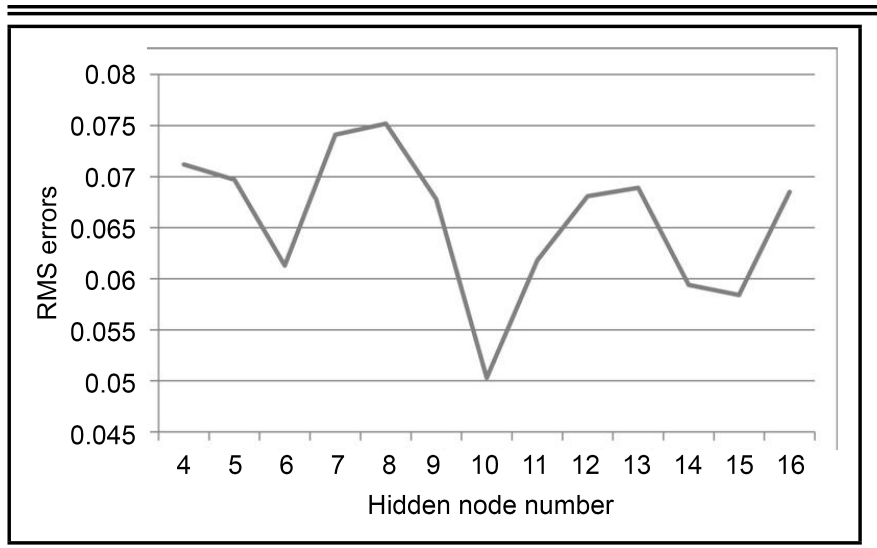

Figure 10. RMS errors with respect to node numbers in the hidden layer.

interval of each vehicle noise is taken as inputs, thus the input node number is 21 . For training a network with $n$ signals, the dimension of the input matrix is extended to $21 \times n$. In this paper, 33 left-ear noise signals measured under the eleven working conditions are taken from the vehicle noise database, i.e., $n=33$. The network output is set to the total roughness at that corresponding moment, which has been calculated in Section 3, thus the output node number is 1 . Accordingly, the output matrix has a dimension of $1 \times 33$. Node number $H_{n}$ of the hidden layer is estimated by the following empirical formula, ${ }^{37}$

$$
H_{n}=\sqrt{I_{n}+O_{n}}+C,
$$

where, $I_{n}$ and $O_{n}$ are the node numbers in the input and output layers, and $C$ is a constant, $C=1-10$. One may calculate that $H_{n}$ equals to 4-16. To find an optimal $H_{n}$ value, a set of training tests was carried out by assuming $H_{n}$ values from 4 to 16 . The averaged RMS errors of ten tests are shown in Figure 10. It can be seen that, with the $H_{n}$ increasing, the errors of predicted results are fluctuant and reach a minimum value at $H_{n}=10$. Thus, the node number of the hidden layer was set to ten in this present work. In addition, the LevenbergMarquardt (LM) algorithm was adopted and used in the ANN training, ${ }^{37}$ due to rapid convergence in solving nonlinear least squares problems.

\subsection{WPD-ANN-ARE Training and Verification}

The ANN training procedure is conducted by a Matlab program. To check the robustness of the ANN structure, the normalized noise samples are divided into three sets for network training, validation, and testing. ${ }^{38}$ The training set is used for learning, which is to fit the ANN weights. The validation set is used to tune the ANN architecture. The test set is used only to assess the performance of the fully ANN. The training and validation sets are defined by 33 left- and right-ear noise signals, and the test set consists of 33 signals that are randomly taken from the vehicle noise database. The robust performance of the WPD-ANN-ARE model is shown in Figure 11. It can be seen that the MSEs of the training, validation and test sets are rapidly decreased within fourteen epochs. The network tends to stabilize at the sixth epoch, and the MSE values reach 0.09 at the eighth epoch. This implies that both the ANN structure and the selected parameters are effective and feasible for ARE of vehicle noise. To verify the WPD-ANN-ARE, furthermore, an original noise signal was randomly selected from the vehicle

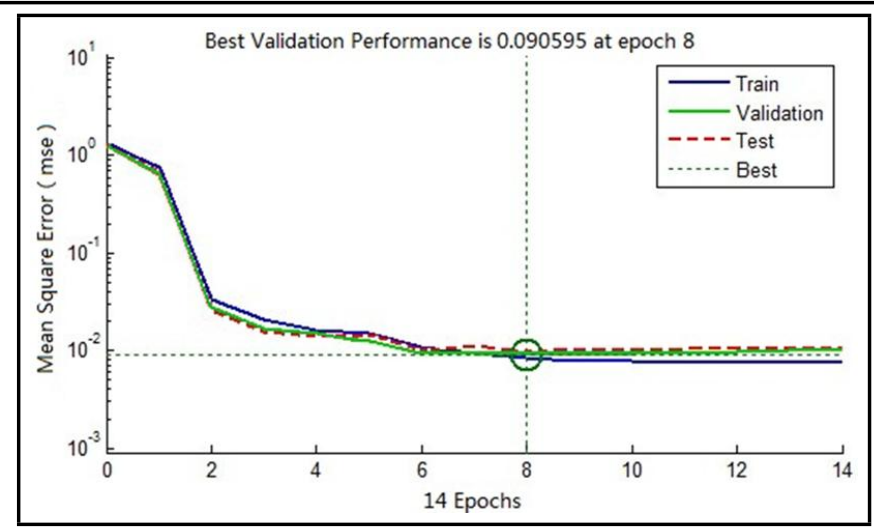

Figure 11. Robust performance of the WPD-ANN-ARE model.

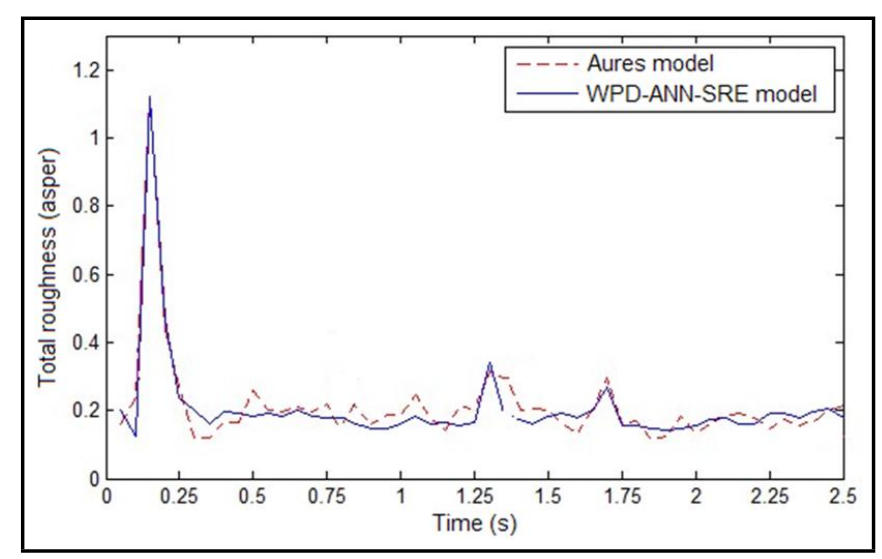

Figure 12. Evaluated comparisons of the roughness results from the WPDANN-ARE and Aures models

noise database and fed to the new model for roughness prediction. Then, the comparison was made between the new model and the Aures model, as shown in Figure 12. The selected vehicle noise was measured at the driver position under the emergency braking condition (stopping from $80 \mathrm{~km} / \mathrm{h}$ ), which is one of the representative conditions. It can be seen that the WPDANN-ARE result is very close to that from the Aures model, especially for the roughness values above 0.3 asper. The mutation of the auditory roughness is predicted by the new model, which shows good characteristic in tracking the extreme values in the time domain, thus able to capture the main sensations of auditory roughness over time. In view of the results, the MSE of the new model result is 0.0427 , and the maximum error of the predicted total roughnesses of the noise signals is 8.56 percent, which can meet the engineering requirements (less than 10 percent). The above comparisons suggest a good accuracy of the WPD-ANN-ARE model in roughness evaluation of vehicle noise. It should be mentioned that there is no any standardized method for ARE till now. The Aures model is the relative authority and has been widely accepted by the international academic community which is the reason why it is adopted as a reference for WPD-ANN-ARE model verification in this paper.

\section{CONCLUSIONS}

This paper presents a new technique named WPD-ANNARE model, which is developed by combining the WPD and ANN, for ARE of vehicle noise. Considering the characteristics of human auditory perception, a WPD-based model with 
21 critical bands is built to extract the noise features. Taking the extracted roughness feature matrices defined by modulation index as inputs and the calculated roughness from the psychoacoustic model as outputs, a three-layer ANN model is designed and trained by the BP algorithm for ARE of noises. The results show that the WPD-based model is effective for feature extraction of nonstationary vehicle noises, and the WPDANN-ARE results are in agreement with those from the conventional roughness model. This implies that the WPD-ANNARE model is accurate enough to map a nonstationary vehicle noise to its roughness, and may be regarded as a good substitute for complex psychoacoustic models in vehicle SQE engineering. In applications, the newly proposed technique can be used to estimate sound quality of vehicles, and may be extended to other noise signals for ARE in engineering.

\section{AKNOWLEDGEMENTS}

This work was supported by National Natural Science Foundation of China (No. 51675324), partly supported by Natural Science Foundation of Shanghai (No. 14ZR1418600).

\section{REFERENCES}

1 Mongelli, Domenico Walter Edvige. An integrated prediction model for traffic noise in an urban area, IEEE International Conference on Service Operations and Logistics, and Informatics IEEE, 198-204, (2013). https://dx.doi.org/10.1109/SOLI.2013.6611409

2 Murata, H., Tanaka, H., Takada, H., Ohsasa, Y. Sound quality evaluation of passenger vehicle interior noise, SAE Technical Paper, 931347, 675-681, (1993). https://dx.doi.org/10.4271/931347

3 Miskiewicz, A., Letowski, T. Psychoacoustics in the automotive industry, Acta Acustica united with Acustica, 85(5), 645-648, (1999).

4 Shin, S. H., Ih, J. G., Hashimoto, T., Hatano, S. Sound quality evaluation of the booming sensation for passenger cars, Applied Acoustics, 70(2), 309-32, (2009). https://dx.doi.org/10.1016/j.apacoust.2008.03.009

5 Zwicker, E., Fastl, H. Psychoacoustics, facts and models, Springer Verlag, Berlin, (1990).

6 Fastl, H. The psychoacoustics of sound-quality evaluation, Acta Acustica united with Acustica, 83(5), 754-764, (1997).

7 Aures, W. A procedure for calculating auditory roughness, Acustica, 58(5), 268-281, (1985).

8 AcousticsMethod for calculating loudness level, ISO 532B:1975, International Organization for Standardization, Geneva, Switzerland, (1975).

9 Measurement technique for the simulation of the auditory sensation of sharpness, DIN 45692, Beuth Verlag GmbH, Berlin, (2009).

10 Helmholtz, H. V. Die Lehre von den Tonempfindungen (The Sensations of Tone), Wissentschaftliche Buchgesellschaft, Darmstadt, unverranderte Auflage, (1862).
11 Stolyarov, U. I., Chisttovich, I. A. Amplitude-frequency characteristics and threshold arrangements of an auditory envelope processing model, Human Physiology, 372, 5559, (1977).

12 Fastl, H. Roughness and temporal masking patterns of sinusoidally amplitude modulated broadband noise, Psychophysics and Physiology of Hearing, 1977, 403-414, (1977).

13 Vassilakis, P. N. Perceptual and physical properties of amplitude fluctuation and their musical significance, Los Angeles: University of California, (2001).

14 Sottek, R. Modelle zur Signalverarbeitung im menschlichen Gehr (Models for signal processing in the human auditory system), Dissertation, TU Aachen, (1993).

15 Daniel, P., Weber, R. Psychoacoustical roughness: implementation of an optimized model, Acta Acustica united with Acustica, 83(1), 113-123, (1997).

16 Wang, Y. S., Shen, G. Q., Guo, H., Tang, X. L., Hamade, T. Roughness modelling based on human auditory perception for sound quality evaluation of vehicle interior noise, Journal of Sound and Vibration, 332(16), 3893-3904, (2013). https://dx.doi.org/10.1016/j.jsv.2013.02.030

17 Hoeldrich, R., Pflueger, M. A parametrized model of psychoacoustical roughness for objective vehicle noise quality evaluation, Journal of the Acoustical Society of America, 105(2), 1371, (1999). https://dx.doi.org/10.1121/1.426489

18 Hashimoto, T. Sound quality approach on vehicle interior and exterior noise - Quantification of frequency related attributes and impulsiveness, Journal of Acoustical Society of Japan (E), 21(6),: 337-340, (2000). https://dx.doi.org/10.1250/ast.21.337

19 Lee, S. K., Kim, B. S., Park, D. C. Objective evaluation of the rumbling sound in passenger cars based on an artificial neural network, Proceedings of the Institution of Mechanical Engineers, Part D: Journal of Automobile Engineering, 219(4), 457-468, (2005). https://dx.doi.org/10.1243/095440705X11112

${ }^{20}$ Lee, S. K., Kim, H. W., Na, E. W. Improvement of impact noise in a passenger car stylizing sound metric based on wavelet transform, Journal of Sound and Vibration, 329(17), 3606-3619, (2010). https://dx.doi.org/10.1016/j.jsv.2010.03.001

21 Wang, Y. S., Lee, C.-M., Kim, D.-G., Xu, Y. Soundquality prediction for nonstationary vehicle interior noise based on wavelet pre-processing neural network model, Journal of Sound and Vibration, 299(4), 933-947, (2007). https://dx.doi.org/10.1016/j.jsv.2006.07.034

22 Huang, H. B., Li, R. X., Xiao Huang, R., Yang, M. L., Ding, W. P. Sound quality evaluation of vehicle suspension shock absorber rattling noise based on the WignerVille distribution, Applied Acoustics, 100, 18-25, (2015). https://dx.doi.org/10.1016/j.apacoust.2015.06.018 
23 Huang, H. B., Huang, X. R., Li, R. X., Lim, T. C., W. P. Ding, W. P. Sound quality prediction of vehicle interior noise using deep belief networks, Applied Acoustics, 113, 149-161, (2016). https://dx.doi.org/10.1016/j.apacoust.2016.06.021

24 Yoon, J. H., Yang, I. H., Jeong, J. E., Park, S. G., Oh, J. E. Reliability improvement of a sound quality index for a vehicle HVAC system using a regression and neural network model, Applied Acoustics, 73, 1099-1103, (2012). https://dx.doi.org/10.1016/j.apacoust. 2012.02.018

25 Huang, H. B., Huang, X. R., Li, R. X., Lim, T. C., Ding, W. P. Identification of vehicle suspension shock absorber squeak and rattle noise based on wavelet packet transforms and a genetic algorithm support vector machine, Applied Acoustics, 113, 137-148, (2016). https://dx.doi.org/10.1016/j.apacoust.2016.06.016

26 Liang, J., Sun, Q., Lu, Y. P. Research on Sound Quality Annoyance Evaluation for Vehicle Interior Noise during Acceleration, Journal of Automotive Technology, 3, 20-25, (2010). https://dx.doi.org/0.3969/j.issn.10003703.2010.03.006

27 Acoustics - Measurement of noise inside motor vehicles, ISO 5128: 1980, International Organization for Standardization, Geneva, Switzerland, (1980).

28 Chatterley, J. J., Boone, A. J., Blotter, J. D., Sommerfeldt, S. D., Lago, T. L. Sound-quality analysis of sewing machines, Journal of the Acoustical Society of America, 116(4), 2591-2600, (2004). https://dx.doi.org/10.1121/1.4785338

29 Luc, M., Immerseel, V., Martens, J. P. Pitch and voiced/unvoiced determination with an auditory model, Journal of the Acoustical Society of America,91(6), 35113526, (1992). https://dx.doi.org/10.1121/1.402840

30 Liu, B., Riemenschneider, S., Xu, Y. Gearbox fault diagnosis using empirical mode decomposition and Hilbert spectrum, Mechanical Systems and Signal Processing, 20(3), 718-734, (2006). https://dx.doi.org/10.1016/j.ymssp.2005.02.003

31 Guo, D., Peng, Z. K. Vibration analysis of a cracked rotor using Hilbert-Huang transform, Mechanical Systems and Signal Processing, 21(8), 3030-3041, (2007). https://dx.doi.org/10.1016/j.ymssp.2007.05.004

32 Cohen, L. Time - Frequency Analysis, Prentice-Hall, Englewood Cliffs, NJ, USA, (1995).

33 Hubbard, B. The World According to Wavelets: The Story of a Mathematical Technique in the Making, Wellesley, USA, (1995).
34 Wang, Y. S. Sound quality estimation for nonstationary vehicle noises based on discrete wavelet transform, Journal of Sound and Vibration, 324(3), 1124-1140, (2009). https://dx.doi.org/10.1016/ j.jsv.2009.02.034

35 Mckeown, J. J., Stella, F., Hall, G. Some numerical aspects of the training problem for feed-forward neural Nets, Neural Networks, 10(9), 1455-1463, (1997). https://dx.doi.org/10.1016/S0893-6080(97)00015-4

36 Hamid, N. A., Nawi, N. M., Ghazali, R., Salleh, M. N. M. Solving local minima problem in back propagation algorithm using adaptive gain, adaptive momentum and adaptive learning rate on classification problems, International Journal of Modern Physics, 9, 448-455, (2012). https://dx.doi.org/10.1142/S2010194512005533

37 Jiang, H., Jia, S., Lai, G. Fault diagnosis of marine main engine based on BP neural network, 8th International Conference on Reliability, Maintainability and Safety, 822-825, (2009). https://dx.doi.org/10.1109/ICRMS.2009.5270075

38 Ripley, B. D. Pattern Recognition and Neural Networks, Cambridge University Press, Cambridge, UK, (1996).

39 Morlet, J., Arens, G., Fourgeau, E., Giard, D. Wave propagation and sampling theory - part I: complex signal and scattering in multilayered media, Geophysics, 47(2),: 203, (1982). https://dx.doi.org/10.1190/1.1441328

40 Xiang, J. W., Chen, X. F., He, Z. J., Dong, H. B. The construction of $1 \mathrm{D}$ wavelet finite elements for structural analysis, Computational Mechanics, 40(2), 325-339, (2007). https://dx.doi.org/10.1007/s00466-006-0102-5

41 Zuo, H., Yang, Z., Chen, X., Xie, Y., Miao, H. Analysis of laminated composite plates using wavelet finite element method and higher-order plate theory, Composite Structures, 131, 248-258, (2015). https://dx.doi.org/10.1016/j.compstruct.2015.04.064

42 Zhang, X., Gao, R.X., Yan, R., Chen, X., Sun, C., Yang, Z. Multivariable wavelet finite element-based vibration model for quantitative crack identification by using particle swarm optimization, Journal of Sound and Vibration, 375, 200216, (2016). https://dx.doi.org/10.1016/j.jsv.2016.04.018

43 Cohen, A. Numerical Analysis of Wavelet Methods, Elsevier Press, Amsterdam, Holland, 2003.

44 Shen, W., Li, D., Zhang, S., \& Ou, J., Analysis of wave motion in one-dimensional structures through fastfourier-transform-based wavelet finite element method, Journal of Sound and Vibration, 400, 369-386, (2017). https://dx.doi.org/10.1016/j.jsv.2017.03.014 